\title{
ANÁLISIS DE INTERACCIONES EN FORO Y CHAT: CONSOLIDACIÓN DE GRUPO Y LIDERAZGO COMUNICATIVO EN UN CURSO DE LÓGICA MATEMÁTICA ${ }^{1}$
}

\author{
(ANALYSIS OF INTERACTIONS IN FORUM AND CHAT: CONSOLIDATION OF GROUP AND \\ COMMUNICATIVE LEADERSHIP IN A MATHEMATICAL LOGICS COURSE)
}

Luis Facundo Maldonado Granados

Linda Alejandra Leal Urueña

Milcon Montenegro Gamba

Universidad Nacional Abierta y a Distancia, UNAD (Colombia)

\section{RESUMEN}

Este artículo analiza la dinámica de comunicación en foro y chat en un ambiente de aula digital en un curso de lógica matemática. Los registros de las sesiones de foro y chat se procesaron mediante un protocolo de análisis. Se elaboran grafos para visualizar la estructuración de los grupos y se calcularon los índices de liderazgo global, individual, de grupo y efectivo de los participantes. De acuerdo con los resultados obtenidos, no hay diferencias relacionadas con la concentración del grupo en la solución de problemas o con la frecuencia de mensajes de clima social atribuibles a los escenarios. La diferencia más significativa entre los escenarios de foro y chat es el índice de liderazgo del tutor.

Palabras clave: Interacción sincrónica, interacción asincrónica, foro, chat, liderazgo, consolidación de grupo.

\begin{abstract}
ABSTRAT
This article analyses the dynamics of communication in the digital classroom environment of a mathematical logic course. Records of the forum and chat sessions were processed using an analysis protocol with different methodological, problem solving and social climate categories. The group structure was graphed and indices of global, individual, group, and effective leadership were calculated. No differences were found with respect to concentration of the group on problem-solving, frequency of messages related to social climate, or scenarios used. The most outstanding difference between the forum and chat scenarios was the index of tutor leadership.
\end{abstract}


Keywords: synchronous interaction, asynchronous interaction, forum, chat, leadership, group consolidation.

Hablamos de sincronía cuando tenemos al menos dos procesos que en un intervalo de tiempo establecen entre sí relaciones de complementariedad e interacción (Sowa, 2000). Habrá mayor sincronía en la medida en que el intervalo de tiempo en el que se establece el vínculo entre los procesos sea más reducido.

En la comunicación pedagógica, entendemos sincronía como un conjunto de procesos de regulación mutua generados por agentes que se comunican y que, en tiempos relativamente cortos, entran en interacción. En los escenarios digitales de aprendizaje, el chat, al exigir menos tiempo de espera para la integración de los procesos de emisión y recepción de la comunicación pedagógica, es más sincrónico que el foro, pero aunque en este último el tiempo entre la emisión del mensaje y la recepción de la respuesta es mayor debe garantizarse siempre la integración entre los dos procesos, de tal manera que la total asincronía no se cumple, pues no se completaría el ciclo de la comunicación.

Las diferencias de sincronía en estos dos escenarios nos hacen suponer que en el foro, el participante puede dar un tratamiento más cuidadoso de la discusión y resolver con mayor eficacia problemas complejos; $y$, que el chat, puede considerarse hipotéticamente como más favorable a la consolidación de grupos debido a la inmediatez de la comunicación, que posibilita el intercambio de saludos y el interés por el interlocutor.

En cuanto a la incorporación de estudiantes a los grupos de discusión, McFadzean y McKenzie (2001) encontraron que cuando los miembros del grupo se perciben a sí mismos como en un grupo heterogéneo, los comentarios son escritos con mayor cuidado con el fin de asegurar que sean entendidos como es deseado, activando la participación para explicar puntos de vista con más deliberación.

Adicionalmente, el estatus de los estudiantes afecta la percepción de liderazgo en un grupo. Un gran número de acciones correctas relativas a otros en un grupo, puede ayudar a la afirmación individual de liderazgo sobre la base del éxito en la solución de problemas. Una cantidad de interacciones individuales con el grupo, particularmente el número de contribuciones y repeticiones, puede facilitar la solución de problemas independientemente de la precisión individual y, por lo tanto, incide en la percepción como líder de un grupo (Ming, 2000). 
En esta investigación, el liderazgo se estudió desde la perspectiva comunicativa, mediante el análisis del número de mensajes y su dirección, independientemente de su precisión. El contenido de los mensajes se analizó para comprobar algunas hipótesis respecto de la incidencia de la sincronía de las interacciones en la dedicación de los grupos a la solución de problemas o a la consolidación de relaciones interpersonales.

Las estrategias empleadas para analizar los efectos de la sincronía de la comunicación y de la distribución de grupos, de acuerdo con el nivel de conocimientos previos, sobre la consolidación de grupos en ambientes digitales de aprendizaje fueron: a) el estudio de las frecuencias de los tipos de mensajes emitidos en las sesiones de foros y chats, según un sistema de categorización, b) la representación de las interacciones en las sesiones de foros y chats en forma de grafos dirigidos, y c) el cálculo de índices de liderazgo total y efectivo.

\section{DISEÑO METODOLÓGICO}

Los estudiantes de un curso de lógica matemática se distribuyeron en dos grupos, de treinta estudiantes cada uno, utilizando como criterio el nivel de conocimientos previos sobre la asignatura. El primer grupo estuvo integrado por estudiantes con conocimientos previos similares, y el otro, por estudiantes con niveles de conocimientos previos heterogéneos. Para efectos de la selección, se diseñó y aplicó una prueba de entrada.

Una vez hecha esta distribución, cada uno de los estudiantes de los grupos se asignó aleatoriamente a uno de dos subgrupos: uno que usó como mecanismo de comunicación pedagógica el foro y otro que usó el chat.

\section{Definición de categorías de análisis de foros y chats}

Para analizar los registros digitales de las sesiones de foros y chats realizadas durante el desarrollo del curso, se tomó como referente el trabajo previo del grupo con ontologías para el análisis de chats (Maldonado, Leal, Montenegro, Pérez y Lineros, 2005). Esta ontología, analiza el proceso de solución de problemas, a partir de un trabajo conceptual y empírico, y define tres categorías de mensajes según su contenido: Aspectos sustanciales de la solución del problema; aspectos metodológicos o mensajes relacionados con el orden de las acciones para poder resolver el problema; y clima social de la participación. 


\begin{tabular}{|c|c|c|}
\hline $\begin{array}{l}\text { Aspectos sustanciales de la } \\
\text { solución del problema }\end{array}$ & $\begin{array}{l}\text { Aspectos de orden } \\
\text { metodológico }\end{array}$ & Clima social de la participación \\
\hline $\begin{array}{l}\text { Introducción a la formulación del } \\
\text { problema }\end{array}$ & $\begin{array}{|lll|}\begin{array}{l}\text { Recordatorio (del tema, del } \\
\text { objetivo) }\end{array} & \\
\end{array}$ & Saludo Retórico \\
\hline Formulación del problema & Moción de orden & Saludo Motivador de la comunicación \\
\hline Reformulación del problema & Sugerencias de acción & $\begin{array}{l}\text { Expresión motivadora para continuar } \\
\text { proceso }\end{array}$ \\
\hline Objetivo principal & $\begin{array}{l}\text { Respuesta positiva a sugerencia } \\
\text { de acción }\end{array}$ & Valoración de la actividad \\
\hline Objetivo parcial & \begin{tabular}{|l|}
$\begin{array}{l}\text { Observaciones de orden técnico } \\
\text { (manejo técnico del ambiente) }\end{array}$ \\
\end{tabular} & Expresión de cortesía \\
\hline $\begin{array}{l}\text { Aporte de información para la } \\
\text { solución del problema }\end{array}$ & \begin{tabular}{|l}
$\begin{array}{l}\text { Recapitulación de temas } \\
\text { anteriores }\end{array}$ \\
\end{tabular} & Comentarios personales \\
\hline \begin{tabular}{|l}
$\begin{array}{l}\text { Sugerencia de alternativa de } \\
\text { solución }\end{array}$ \\
\end{tabular} & Solicitud de recapitulación & \\
\hline \begin{tabular}{|l|} 
Refuta alternativa de solución \\
\end{tabular} & Respuesta de recapitulación & \\
\hline $\begin{array}{l}\text { Complementa alternativa de solución } \\
\text { (acepta y amplía) }\end{array}$ & Pregunta de control de lectura & \\
\hline $\begin{array}{l}\text { Aprobación de alternativa de } \\
\text { solución }\end{array}$ & $\begin{array}{l}\text { Respuesta negativa al control de } \\
\text { lectura }\end{array}$ & \\
\hline Pregunta de aclaración & $\begin{array}{l}\text { Respuesta positiva al control de } \\
\text { lectura }\end{array}$ & \\
\hline Respuesta de aclaración & $\begin{array}{l}\text { Respuesta de ejecución parcial } \\
\text { al control de lectura }\end{array}$ & \\
\hline \begin{tabular}{|l|} 
Pregunta de opinión \\
\end{tabular} & Disculpar por omisión de tareas & \\
\hline Respuesta de opinión & $\begin{array}{|lll|}\begin{array}{l}\text { Confirmación } \\
\text { información }\end{array} & \text { envío de } \\
\end{array}$ & \\
\hline Solicitud de síntesis & $\begin{array}{l}\text { Confirmación de recepción de } \\
\text { información }\end{array}$ & \\
\hline $\begin{array}{l}\text { Síntesis o integración de alternativas } \\
\text { de solución }\end{array}$ & Solicitud de información & \\
\hline
\end{tabular}

Tabla 1. Categorías de mensaje según su contenido

\section{Frecuencia de mensajes emitidos por categorías e hipótesis}

Con los resultados del proceso de clasificación de mensajes sobre los registros de las sesiones de foro y chat, se calcularon las frecuencias de mensajes emitidos en cada una de las categorías del protocolo de análisis con el fin de analizar diferencias entre los escenarios de comunicación foro y chat. 
Como hipótesis de investigación se plantearon las siguientes:

Hipótesis 1. El foro, al procesar mensajes a una velocidad menor, favorece un tratamiento más cuidadoso de la discusión y es más favorable a la solución de problemas

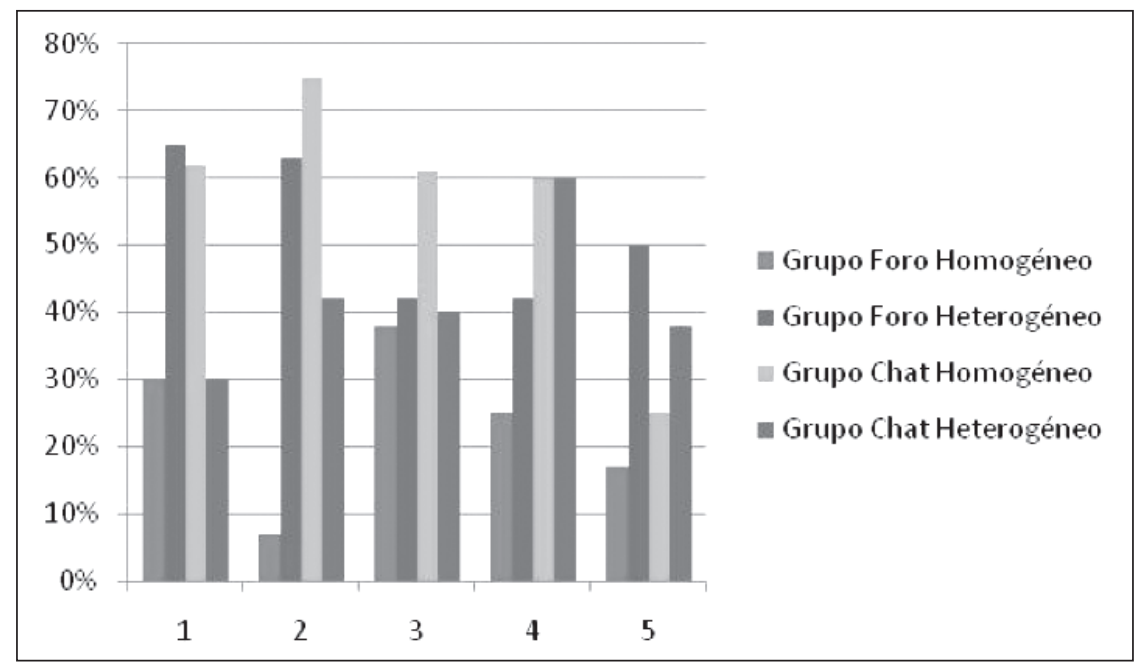

Ilustración 1. Evolución porcentual de mensajes relacionados con aspectos sustanciales de la solución del problema

La ilustración 1, presenta la evolución porcentual de mensajes emitidos afines con aspectos relacionados con la solución del problema en los cuatro grupos del curso. En ella se ve que los porcentajes más bajos de mensajes emitidos sobre la solución del problema se presentan en el grupo foro homogéneo y en el grupo foro heterogéneo la tendencia es decreciente. Los porcentajes más altos de mensajes relacionados con la solución de problemas se presentan en el grupo de chat homogéneo.

De acuerdo con estos datos, el escenario de comunicación de foro o chat no incide de manera consistente en la dedicación del grupo en la solución de problemas.

Hipótesis 2. El chat puede considerarse como más favorable a la consolidación de grupos, por lo cual los grupos de chat dedicarán mayor parte de la sesión a mensajes de clima social. 


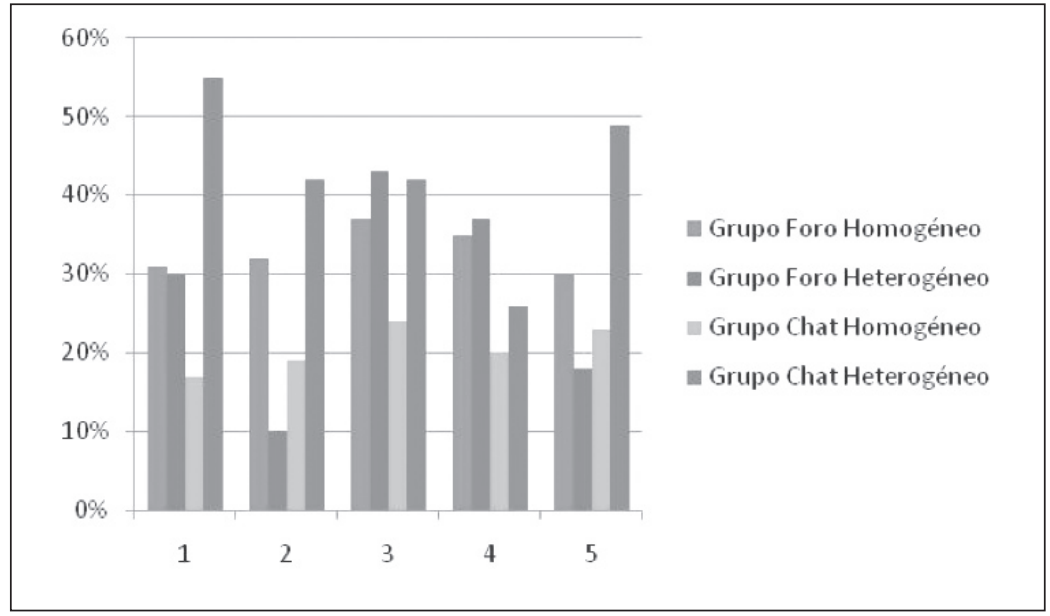

Ilustración 2. Evolución porcentual de mensajes relacionados con clima social de la participación

La ilustración 2, presenta la evolución porcentual de mensajes emitidos relacionados con el clima social de la participación. En la gráfica se aprecia que los porcentajes más altos relacionados con el clima social de la participación se encuentran en el grupo chat heterogéneo, seguido por el grupo de foro homogéneo. En el grupo chat homogéneo presenta el menor porcentaje de mensajes de este tipo durante el desarrollo del curso.

En relación con el escenario de comunicación de foro o chat, no se evidencia ninguna incidencia consistente de este con una mayor o menor frecuencia de emisión de mensajes relacionados con el clima social.

\section{Conceptos para la definición del liderazgo en la comunicación pedagógica}

Con el fin de comprender las dinámicas de comunicación e interacción en los escenarios de foro y chat, se propone el siguiente sistema de conceptos e índices para estudiar el liderazgo desde la perspectiva comunicativa, mediante el análisis de la cantidad y dirección de las contribuciones individuales con el grupo en el proceso de la solución de problemas.

Sesión (o reunión interactiva). Está delimitada por coordenadas temporales, a saber: hora y fecha de inicio y hora y fecha de cierre. En el caso del foro se abre en un día y hora y minuto específicos y se cierra en otro día y hora y minuto específico. El Chat normalmente se inicia y termina en el mismo día. 
Proceso Interactivo: Conjunto de sesiones de referencia organizadas por sucesión temporal. Los cálculos de liderazgo aplicados a una sesión de referencia se utilizan como elementos para la visualización y el cálculo de los indicadores de liderazgo a lo largo de un proceso.

Emisión $\left(E_{i}\right)$ : Definido como el número de mensajes emitidos por un nodo (i) durante una sesión. Se expresa por un número entero.

Nivel de recepción de un nodo $\left(R_{i}\right)$. Cantidad de mensajes recibidos por un nodo (i). Se expresa por un número entero.

Emisión al grupo (Eoo $)_{i}$. Número de mensajes dirigidos por un nodo (i) al nodo "todos" (Foo en las ilustraciones).

Emisión orientada a individuos $\left(E i_{i}\right)$. Total de mensajes dirigidos por un nodo (i) a nodos diferentes al nodo "todos".

Nivel global de emisión de la sesión (Et): Total de mensajes emitidos durante una sesión de referencia. Se expresa por un número entero. Es la sumatoria de las emisiones correspondientes a cada nodo. Por ejemplo, en la ilustración 23, este nivel es de 43. $\mathrm{Et}=\sum_{\mathrm{i}}^{\mathrm{n}}\left(\mathrm{Eoo}_{\mathrm{i}}+\mathrm{Ei}_{\mathrm{i}}\right)$.

Nivel global de recepción de la sesión (Rt). Total de mensajes recibidos por todos los nodos. Se expresa como un número entero, es equivalente a la sumatoria de los mensajes enviados a nodos individuales. $\mathrm{Rt}=\sum_{\mathrm{i}}{ }^{\mathrm{n}}\left(\mathrm{Ei}_{\mathrm{i}}\right)$

Nivel Global de orientación al grupo (Dg). Proporción de mensajes dirigidos por todos los nodos al nodo "todos". Dg $=\sum_{\mathrm{i}}{ }^{\mathrm{n}}\left(\mathrm{Eoo}_{\mathrm{i}}\right) /$ Et.

Nivel Global de orientación individual (Di). Proporción de mensajes dirigidos por todos los todos a nodos individuales o diferentes al nodo "todos". Di = $\sum$ in(Eii)/ Et.

\section{Orientaciones de la emisión de un nodo (o participante)}

Orientación al grupo $\left(O g_{i}\right)$ : Proporción de mensajes dirigidos por un nodo al nodo "todos" (Foo en las ilustraciones). Su formula es:

(1) Ogi $=$ Eooi/Ei. Es un número real con variación entre o y 1 . 
Orientación a individuos: Proporción de mensajes emitidos por un nodo a nodos diferentes al nodo "todos". Su formula es:

(2) $\mathrm{Oi}_{\mathrm{i}}=\mathrm{Ei}_{\mathrm{i}} / \mathrm{E}_{\mathrm{i}}$. Es un número real con variación entre o y 1 .

$\mathrm{Og}_{\mathrm{i}} \mathrm{y} \mathrm{Oi}_{\mathrm{i}}$ son números complementarios; su suma es igual a 1, cuando el nodo ha emitido mensajes. Si un nodo no tiene emisiones, no tiene conexiones gráficas en la representación de la sesión.

\section{Índice de Liderazgo Total $(\mathrm{Lg})$}

Se comprende como la proporción de mensajes emitidos por un individuo (Ei) dividido por el número total de mensajes emitidos durante la sesión o Emisión total (Et).

(3) $\mathrm{Lg}_{\mathrm{i}}=\mathrm{E}_{\mathrm{i}} / \mathrm{Et}=\left(\mathrm{Eoo}_{\mathrm{i}}+\mathrm{Ei}_{\mathrm{i}}\right) /$ Et Es un número real con rango de variación entre o y 1

Este es complementado por los Índices de Liderazgo de Grupo y de Individuos de un nodo (o participante) que se describen a continuación:

(4) $\mathrm{Li}=\mathrm{Ei}_{\mathrm{i}} / \mathrm{Et}$ (Liderazgo de Individuos de un nodo)

(5) $\mathrm{Loo}_{\mathrm{i}}=\mathrm{Eoo}_{\mathrm{i}} / \mathrm{Et}$ (Liderazgo de Grupo de un nodo)

\section{Liderazgo efectivo de un nodo $\left(\mathrm{Ce}_{\mathrm{i}}\right)$}

Es el índice que relaciona la cantidad de mensajes emitidos con la cantidad de mensajes recibidos. Es un modelo similar a una ecuación de capital por intereses: expresa la relación entre el esfuerzo invertido con las respuestas obtenidas y dirigidas al individuo. Es proporcional a la cantidad de respuestas obtenidas e inversamente proporcional a los mensajes emitidos.

$$
\text { (6) } \mathrm{Ce}_{\mathbf{i}}=\left(\mathrm{R}_{\mathbf{i}}-\mathrm{E}_{\mathbf{i}}\right) / \mathrm{E}_{\mathbf{i}}=\left(\mathrm{R}_{\mathbf{i}} / \mathrm{E}_{\mathbf{i}}\right)-1
$$

Entre mayor sea el coeficiente, mayor es la efectividad de liderazgo de un individuo. Si un nodo emite x mensajes, realiza un esfuerzo para obtener respuestas. Se esperaría, al menos una respuesta, de manera que el primer elemento de la formula es la diferencia entre respuestas obtenidas y mensajes enviados. $R_{\mathbf{i}}-E_{\mathbf{i}}$. Esta 
diferencia se distribuye entre el número de mensajes enviados $\left(\mathrm{R}_{\mathrm{i}}-\mathrm{E}_{\mathrm{i}}\right) / \mathrm{E}_{\mathrm{i}}$, de tal manera que se halle la efectividad de cada mensaje.

Si la diferencia $\mathrm{R}_{\mathrm{i}}-\mathrm{E}_{\mathrm{i}}$ es negativa, el participante obtuvo menos respuestas que mensajes enviados. Si es positiva, tuvo un número de respuestas mayor o igual a los mensajes emitidos y la ganancia se distribuye entre los mensajes emitidos.

El liderazgo efectivo se puede analizar en términos de liderazgo efectivo orientado al grupo y liderazgo efectivo orientado a individuos:

(7) $\mathrm{Ce}_{\mathrm{i}}=\left(\mathrm{R}_{\mathrm{i}}-\left(\mathrm{Eoo}_{\mathrm{i}}+\mathrm{Ei}_{\mathrm{i}}\right)\right) / \mathrm{E}_{\mathrm{i}}=\mathrm{R}_{\mathrm{i}} / \mathrm{E}_{\mathrm{i}}-\left(\mathrm{Eoo}_{\mathrm{i}} / \mathrm{E}_{\mathrm{i}}+\mathrm{Ei}_{\mathrm{i}} / \mathrm{E}_{\mathrm{i}}\right)$ Debido a que $\mathrm{E}_{\mathrm{i}}=\mathrm{Eoo}_{\mathrm{i}}+\mathrm{Ei}_{\mathrm{i}}$.

Por ejemplo, un participante que interviene dos veces y obtiene diez respuestas en promedio, tendría mayor liderazgo del que interviene 100 veces y obtiene 102 respuestas.

Es posible evaluar la sesión con el índice de liderazgo efectivo si se aplica estadística descriptiva al conjunto de los individuos. De esta manera se obtiene el promedio de liderazgo efectivo, la desviación estándar y la comparación de los liderazgos individuales. De esta manera se puede definir que tanto domina un nodo a los demás.

(8) promedio de $\mathrm{CE}_{\mathrm{i}}^{\mathrm{n}}=\sum_{\mathrm{i}}^{\mathrm{n}}\left(\mathrm{Ce}_{\mathrm{i}}\right) / \mathrm{n}$

\section{ANÁLISIS DEL LIDERAZGO COMUNICATIVO EN CHATS Y FOROS}

Como análisis complementario se elaboraron grafos para visualizar la estructuración de los grupos y la dinámica de comunicación e interacción en tres momentos de la evolución del curso. En los grafos, cada nodo corresponde a un participante, siendo el nodo oo la entidad "Todos", que representa al grupo general. En cada nodo se encuentra el número de mensajes recibidos (parte superior), el código asignado al participante (centro) y el número de mensajes emitidos (parte inferior). Los arcos en los grafos fueron etiquetados con el número total de mensajes emitidos.

Las representaciones gráficas construidas se analizaron a partir del cálculo de los índices de liderazgo de los nodos respecto a los demás participantes y al grupo y del 
cálculo del liderazgo efectivo de cada nodo a partir de la relación entre los mensajes recibidos y emitidos.

\section{Grupos que utilizaron como escenario de comunicación el chat}

\section{Grupo Chat Homogéneo}

\begin{tabular}{|c|c|c|c|c|c|c|}
\hline$\frac{(c 00)}{(c 22)}$ & 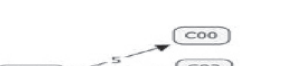 & Nodo & $\mathrm{Lg}$ & $\operatorname{Lg}(00)$ & $\lg (\mathrm{i})$ & $\mathrm{Ce}$ \\
\hline $\begin{array}{l}=(23) \\
=(c 24)\end{array}$ & 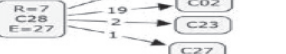 & C02 & 0,25 & 0,19 & 0,06 & 1,11 \\
\hline $\begin{array}{l}-(c 26) \\
C 27\end{array}$ & coo & C27 & 0,04 & 0,02 & 0,02 & $-0,67$ \\
\hline (C32) & $=02)$ & C33 & 0,04 & 0,01 & 0,03 & $-0,73$ \\
\hline & 02 & $\mathrm{C} 28$ & 0,09 & 0,02 & 0,07 & $-0,74$ \\
\hline$=(\cos )$ & 204 & $\mathrm{C} 21$ & 0,07 & 0,02 & 0,05 & $-0,75$ \\
\hline $\begin{array}{l}=c 02 \text { c2 } \\
=c 22\end{array}$ & & $\mathrm{C} 26$ & 0,02 & 0,00 & 0,01 & $-0,80$ \\
\hline $\begin{array}{l}-c 28 \\
-C 00\end{array}$ & $=\frac{C_{24}}{C_{23}}$ & $\mathrm{C} 20$ & 0,04 & 0,00 & 0,03 & $-0,82$ \\
\hline $\begin{array}{l}=\mathrm{CO}^{\circ} \\
=\mathrm{C}_{20}\end{array}$ & $-\frac{c 32}{c o 0}$ & C24 & 0,08 & 0,01 & 0,07 & $-0,84$ \\
\hline$-\frac{(100)}{c_{0102}}$ & $\begin{array}{l}=(\mathrm{COO} 2) \\
=(\mathrm{C22})\end{array}$ & $\mathrm{C} 22$ & 0,13 & 0,02 & 0,12 & $-0,85$ \\
\hline $\begin{array}{l}(02) \\
\frac{220}{21}\end{array}$ & & $\mathrm{C} 23$ & 0,19 & 0,06 & 0,14 & $-0,86$ \\
\hline & (c33) & C32 & 0,06 & 0,01 & 0,05 & $-0,88$ \\
\hline is) & & $\mathrm{CO4}$ & 0,00 & 0,00 & 0,00 & $-1,00$ \\
\hline 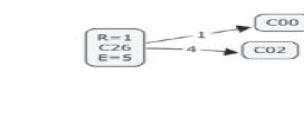 & 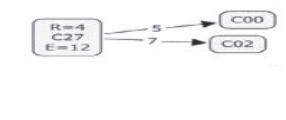 & \multicolumn{5}{|c|}{$\begin{array}{c}\text { Índices de liderazgo total, de individuo } \\
\text { de grupo y efectivo }\end{array}$} \\
\hline
\end{tabular}

Ilustración 3. Sesión de Chat No. 3 Proposiciones y conectores Lógicos - Grupo Homogéneo

La Ilustración 3, muestra que el tutor, nodo Co2, tiene los índices más altos de liderazgo general, de grupo y efectivo. El nodo C23, presenta el mayor valor de liderazgo de individuos (o.14) y el segundo valor en liderazgo global. Los nodos de los estudiantes tienen un liderazgo efectivo negativo, en todos los casos. Si bien la participación se distribuye principalmente entre tres nodos, el tutor es quien tiene mayor tasa de respuesta.

Los pesos de las conexiones muestran que el más alto índice de mensajes se produce hacia el grupo general por parte del tutor, seguido de la comunicación que se establece en relación uno a uno tutor-estudiante. La comunicación entre pares es casi nula. 


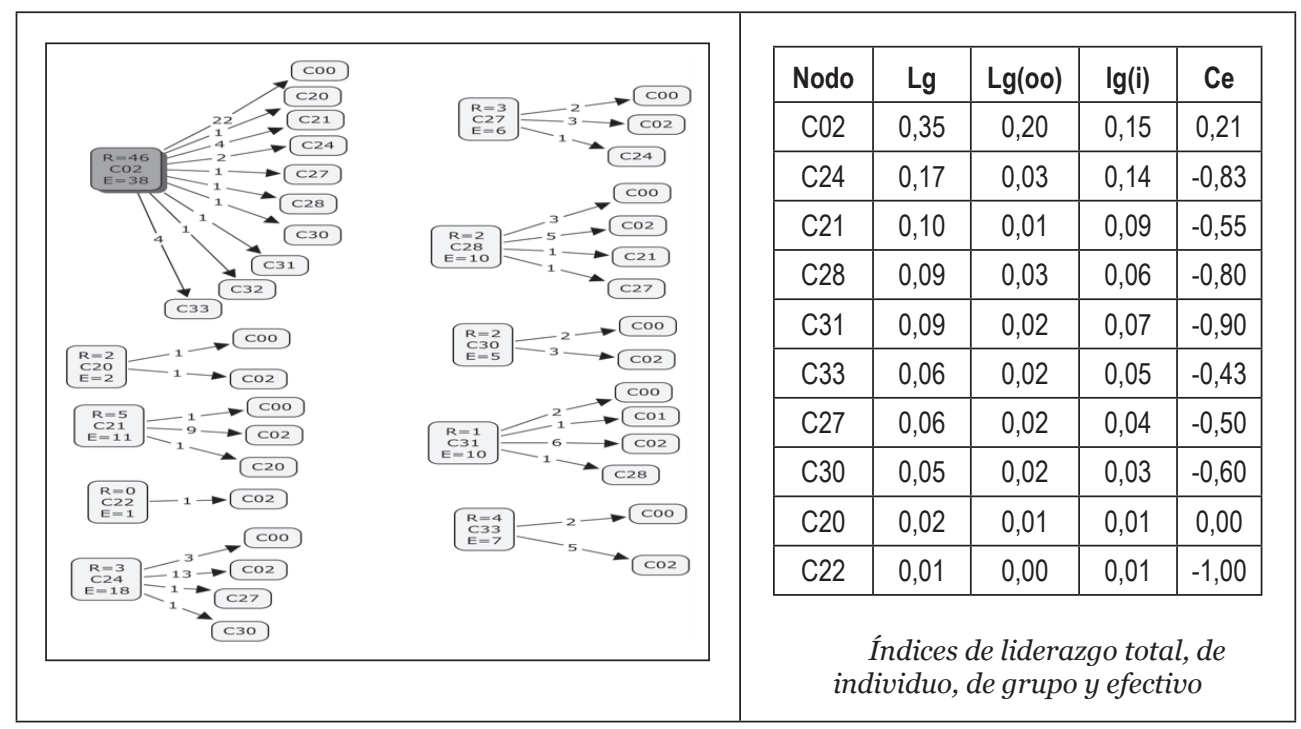

Ilustración 4. Sesión de Chat No. 5 Inferencia Lógica - Grupo Homogéneo

En el grafo de la Ilustración 4, el tutor, nodo Co2, tiene los índices más altos de liderazgo general, de grupo, de individuos y efectivo. El estudiante representado por el nodo C24 presenta un valor de liderazgo de individuos semejante al del tutor y el segundo liderazgo global. Los nodos de los estudiantes tienen un liderazgo efectivo negativo, en todos los casos.

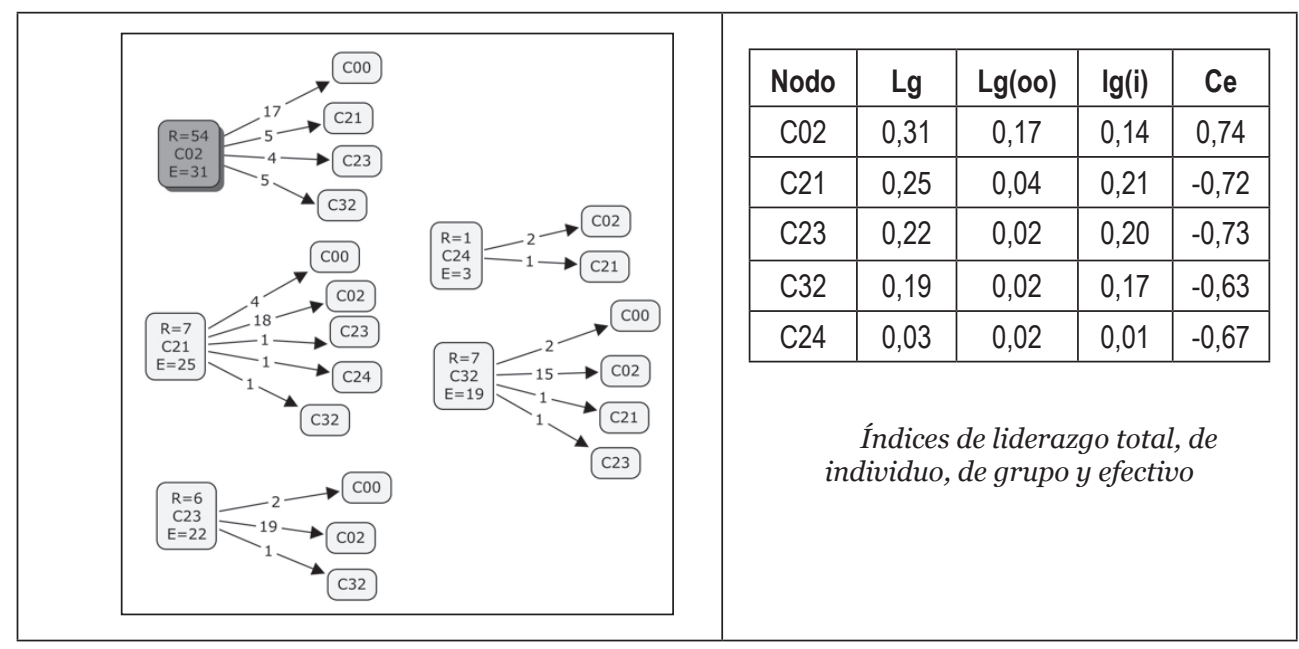

Ilustración 5. Sesión de Chat No. 8. Inferencia Lógica y de predicados - Grupo Homogéneo 
En el grafo de la Ilustración 5, el tutor, nodo Co2, tiene los índices más altos de liderazgo general, de grupo y efectivo. Los estudiantes representados por los nodos C21, C22 y C23 presentaron los valores más significativos en el índice de liderazgo de individuos. El liderazgo tanto global como individual se distribuye homogéneamente entre los participantes, con excepción del C24, que muestra índices relativamente bajos. Los nodos de los estudiantes tienen un liderazgo efectivo negativo, en todos los casos, en contraste con el tutor al cual se dirigen la mayoría de respuestas.

En síntesis, el liderazgo en la comunicación en el grupo de chat homogéneo se caracteriza por:

- El tutor es el único actor del proceso de comunicación que presenta índices de liderazgo efectivo positivos.

- En los casos analizados, el tutor es también quien presenta los mayores índices de liderazgo general y de grupo.

- Algunos estudiantes sobresalen en el liderazgo de individuos, en algunos casos superando al tutor.

\section{Grupo Chat Heterogéneo}

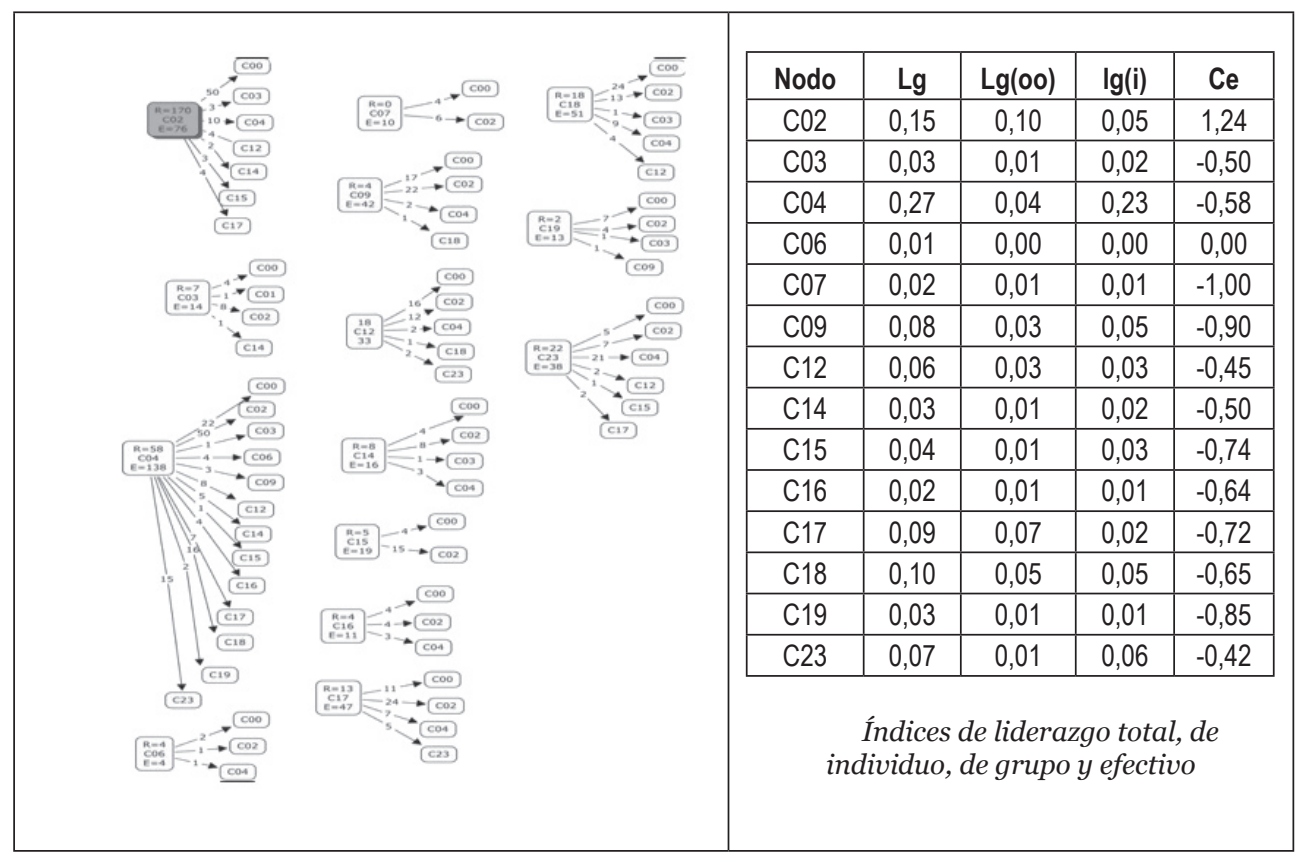

Ilustración 6. Sesión de Chat No. 3 Proposiciones y conectores Lógicos - Grupo Heterogéneo 
En la Ilustración 6 se aprecia, que el tutor, nodo Co2, tiene los índices más altos de liderazgo efectivo y de grupo. El estudiante representado por el nodo Co4 presenta los valores más significativos en los índices de liderazgo general y de individuos. Pese a la participación de este nodo, el nivel de respuesta obtenido tiene una tasa más baja que la del tutor. Los nodos de los estudiantes tienen un liderazgo efectivo negativo, en todos los casos.

ind

Ilustración 7. Sesión de Chat No. 4. Tablas de Verdad - Grupo Heterogéneo

La Ilustración 7, muestra que el tutor, nodo Co2, tiene los índices más altos de liderazgo de grupo y efectivo. El estudiante representado por el nodo Co4 presenta los valores más significativos en los índices de liderazgo general y de individuos. Igual al caso anterior, la tasa de respuesta al tutor es superior a la de los estudiantes Los nodos de los estudiantes tienen un liderazgo efectivo negativo, en todos los casos.

En síntesis, el liderazgo en la comunicación en el grupo de chat heterogéneo se caracteriza por: 
- El tutor es el único actor del proceso de comunicación que presenta índices de liderazgo efectivo positivos.

- En los casos analizados, el tutor es también quien presenta el mayor índice de liderazgo de grupo.

- Uno de los estudiantes sobresale en el liderazgo general, el tutor presenta el segundo valor más alto en este índice.

- El liderazgo entre individuos sobresale especialmente entre los estudiantes, aunque los mensajes emitidos están dirigidos hacia el tutor.

\section{Grupos que utilizaron como escenario de comunicación el foro}

\section{Grupo Foro Homogéneo}
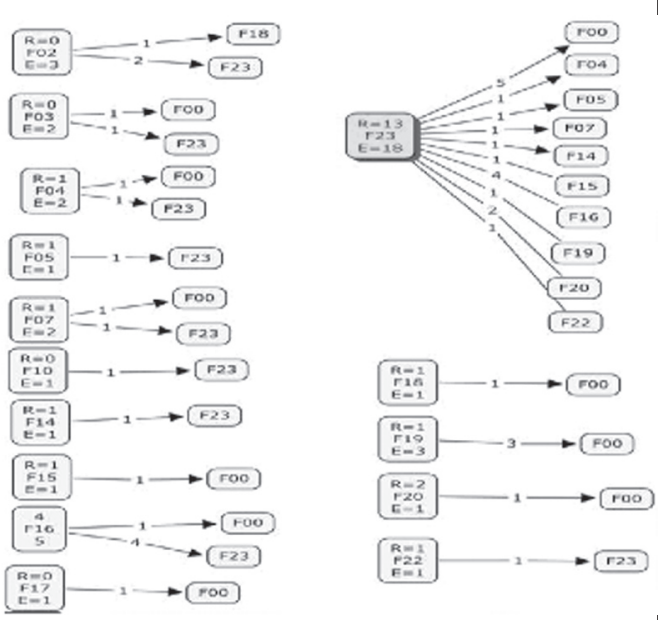

\begin{tabular}{|c|c|c|c|c|}
\hline Nodos & Lg & Lg(00) & lg(i) & Ce \\
\hline F22 & 0,02 & 0,02 & 0,00 & 0,00 \\
\hline F05 & 0,02 & 0,00 & 0,02 & 0,00 \\
\hline F10 & 0,02 & 0,00 & 0,02 & 0,00 \\
\hline F14 & 0,02 & 0,00 & 0,02 & 0,00 \\
\hline F15 & 0,02 & 0,02 & 0,00 & 0,00 \\
\hline F18 & 0,02 & 0,02 & 0,00 & 0,00 \\
\hline F16 & 0,12 & 0,02 & 0,10 & $-0,20$ \\
\hline F23 & 0,44 & 0,12 & 0,32 & $-0,28$ \\
\hline F04 & 0,05 & 0,02 & 0,02 & $-0,50$ \\
\hline F07 & 0,05 & 0,02 & 0,02 & $-0,50$ \\
\hline F19 & 0,07 & 0,07 & 0,00 & $-0,67$ \\
\hline F02 & 0,07 & 0,02 & 0,05 & $-1,00$ \\
\hline F03 & 0,05 & 0,02 & 0,02 & $-1,00$ \\
\hline F17 & 0,02 & 0,02 & 0,00 & $-1,00$ \\
\hline
\end{tabular}

Índices de liderazgo total, de individuo, de grupo y efectivo

Ilustración 8. Sesión de Foro No. 3 Proposiciones y conectores Lógicos - Grupo Homogéneo

La ilustración 8, muestra que el tutor, representado por el nodo F23, tiene los índices más altos de liderazgo general, de grupo y de individuos. Todos los participantes tuvieron un liderazgo efectivo igual a cero o negativo. Esto muestra que el nivel de respuesta de los participantes es bajo y que lograr una participación 
implica un esfuerzo mayor que en la dinámica observada en los grupos de chat homogéneo. El tutor dirige su principal esfuerzo a los individuos en contraste con el chat homogéneo.

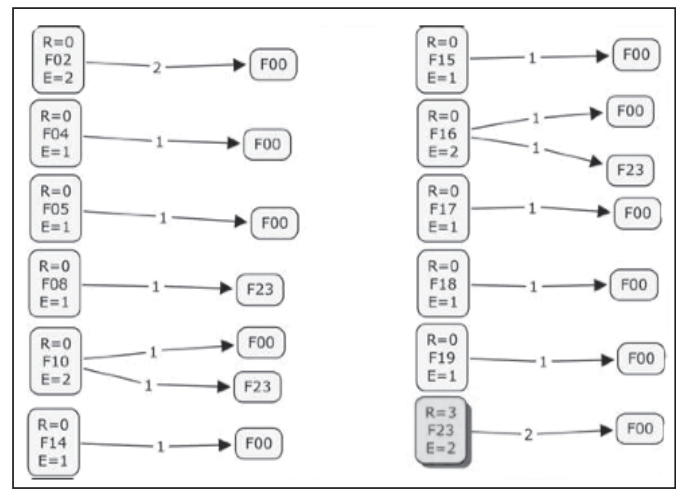

\begin{tabular}{|c|c|c|c|c|}
\hline Nodos & Lg & Lg(oo) & Ig(i) & Ce \\
\hline F23 & 0,13 & 0,13 & 0,00 & 0,50 \\
\hline F02 & 0,13 & 0,13 & 0,00 & $-1,00$ \\
\hline F04 & 0,06 & 0,06 & 0,00 & $-1,00$ \\
\hline F05 & 0,06 & 0,06 & 0,00 & $-1,00$ \\
\hline F08 & 0,06 & 0,00 & 0,06 & $-1,00$ \\
\hline F10 & 0,13 & 0,06 & 0,06 & $-1,00$ \\
\hline F14 & 0,06 & 0,06 & 0,00 & $-1,00$ \\
\hline F15 & 0,06 & 0,06 & 0,00 & $-1,00$ \\
\hline F16 & 0,13 & 0,06 & 0,06 & $-1,00$ \\
\hline F17 & 0,06 & 0,06 & 0,00 & $-1,00$ \\
\hline F18 & 0,06 & 0,06 & 0,00 & $-1,00$ \\
\hline F19 & 0,06 & 0,06 & 0,00 & $-1,00$ \\
\hline
\end{tabular}

Índices de liderazgo total, de individuo, de grupo y efectivo

Ilustración 9. Sesión de Foro No. 5 Inferencia Lógica - Grupo Homogéneo

La ilustración 9 muestra que el tutor, nodo F23, tiene el índice más alto de liderazgo efectivo. El liderazgo general, de grupo y de individuos es compartido con el tutor con algunos estudiantes. El valor más alto de liderazgo de individuos se presenta en el estudiante representado por el nodo F16, quien emitió mensajes para el tutor y para el grupo general. Los nodos de los estudiantes tienen un liderazgo efectivo negativo, en todos los casos. Es de notar que la dirección de los mensajes del tutor se dirigen al grupo y no a individuos, en contraste con la sesión anterior y, correlativamente, los mensajes de los estudiantes se dirigen predominantemente al grupo y el costo de respuesta para los estudiantes es igual a -1,0, lo cual se asocia con una tasa de respuesta baja, generalmente una respuesta. 


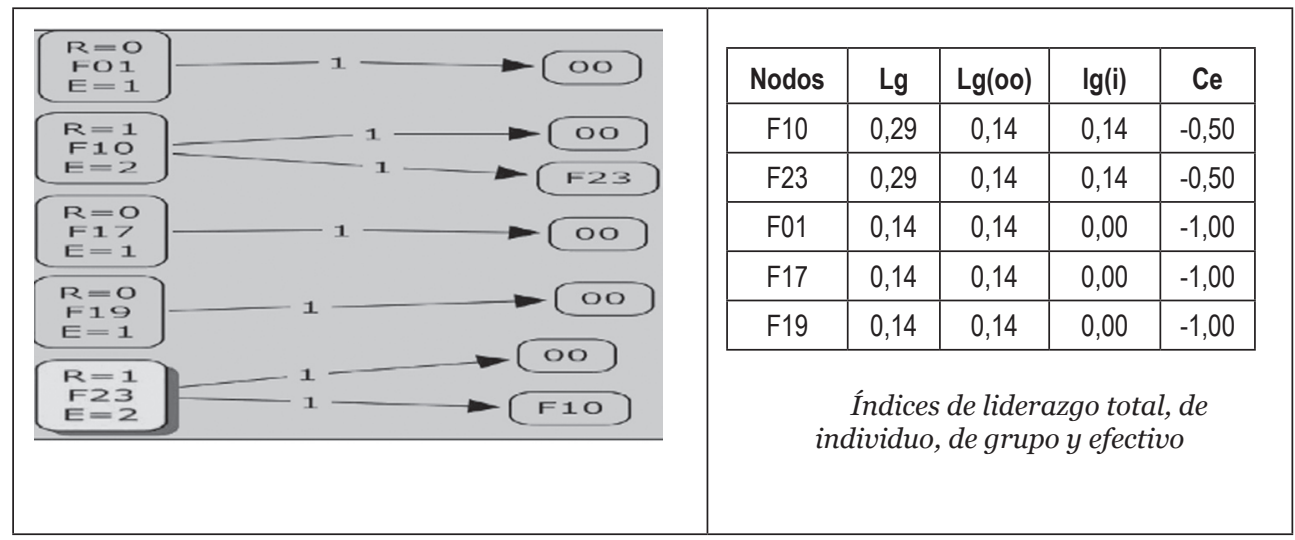

Ilustración 10. Sesión de Foro No. 7 Nombres, predicados y cuantificadores - Grupo Homogéneo

La ilustración 10 muestra que los nodos que representan al tutor (F23) y a uno de los estudiantes (F10) presentan los índices más altos de liderazgo general y de individuos. El liderazgo de grupo presenta el mismo valor en todos los participantes, lo cual indica un nivel de participación distribuido de manera muy homogénea y un nivel de respuesta bajo.

En síntesis, el liderazgo en la comunicación en el grupo de foro homogéneo se caracteriza por:

- En los casos analizados, los valores de liderazgo efectivo son iguales a cero o negativos tanto para el tutor como para los estudiantes, sólo en uno de los casos el tutor presentó liderazgo efectivo positivo.

- Los valores más altos de liderazgo general y de grupo se distribuyen entre el tutor y en algunos de los estudiantes.

- El liderazgo entre individuos sobresale especialmente entre los estudiantes, aunque los mensajes emitidos están dirigidos en la mayoría de los casos hacia el tutor, la interacción entre pares es nula.

- El nivel de respuesta a los mensajes es baja, generalmente cercana a 1. 


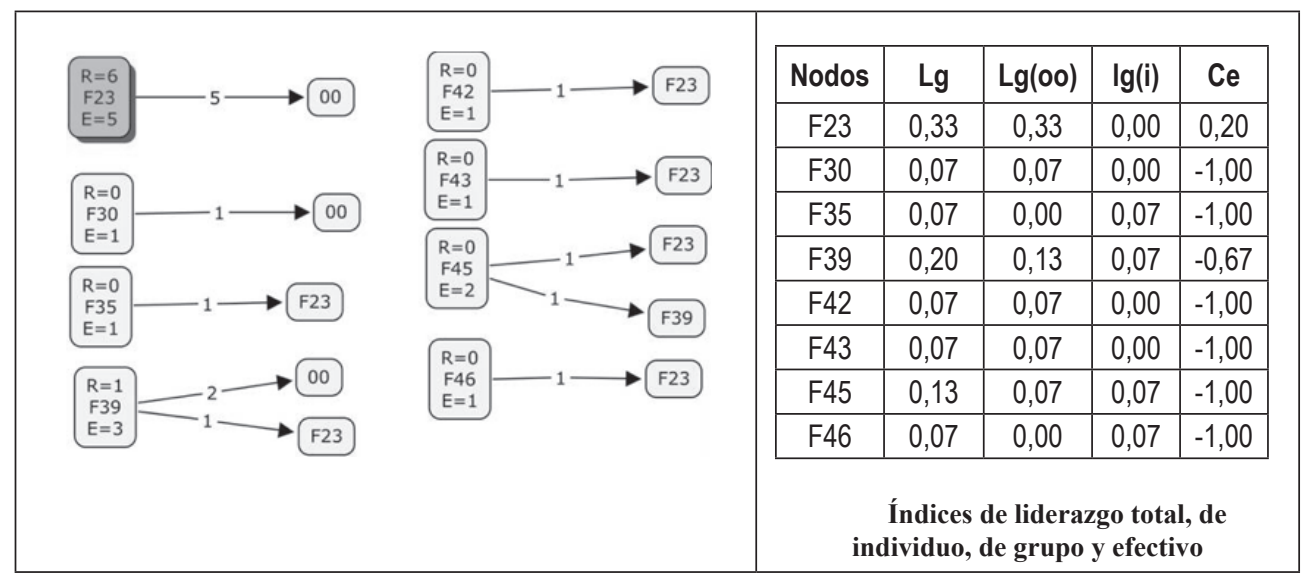

Ilustración 11. Sesión de Foro No. 3 Proposiciones y conectores Lógicos - Grupo Heterogéneo

La ilustración 11, muestra que el tutor, nodo F23, tiene los índices más altos de liderazgo de general, de grupo y efectivo. El liderazgo de individuos es compartido por algunos estudiantes, quienes emiten mensajes dirigidos al tutor. Los nodos de los estudiantes tienen un liderazgo efectivo negativo, en todos los casos.

\begin{tabular}{|c|c|c|c|c|c|c|}
\hline \multirow{6}{*}{$\left.\begin{array}{c|cc}R=0 \\
R=25 \\
E=2\end{array}\right] 2 \longrightarrow$} & \multirow{2}{*}{ 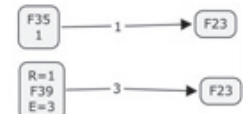 } & Nodos & $\mathrm{Lg}$ & $\operatorname{Lg}(00)$ & $\lg (i)$ & $\mathrm{Ce}$ \\
\hline & & F23 & 0,29 & 0,18 & 0,12 & 0,70 \\
\hline & & F25 & 0,06 & 0,00 & 0,06 & $-1,00$ \\
\hline & {$\left[\begin{array}{l}R=1 \\
F=1 \\
k=-1\end{array}\right]$} & F28 & 0,03 & 0,00 & 0,03 & $-1,00$ \\
\hline & $R=1$ & F30 & 0,06 & 0,03 & 0,03 & $-1,00$ \\
\hline & 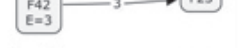 & F31 & 0,06 & 0,00 & 0,06 & $-1,00$ \\
\hline$\underset{\substack{R=0 \\
F 28}}{\longrightarrow} \longrightarrow$ & 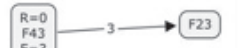 & F32 & 0,03 & 0,00 & 0,03 & 0,00 \\
\hline$\xi=-1$ & & F35 & 0,06 & 0,03 & 0,03 & $-1,00$ \\
\hline 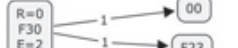 & $\begin{array}{l}\begin{array}{l}2-0 \\
k 45 \\
k=2\end{array} \\
E=2\end{array}-1-$ & F39 & 0,09 & 0,00 & 0,09 & $-0,67$ \\
\hline$R=0$ & & F40 & 0,03 & 0,03 & 0,00 & $-1,00$ \\
\hline $\begin{array}{l}\beta 31 \\
E=2\end{array}$ & $\begin{array}{l}R=1 \\
F=4 \\
F=2 \\
E=2\end{array} \underbrace{-1} \longrightarrow \infty$ & F42 & 0,09 & 0,00 & 0,09 & $-1,00$ \\
\hline 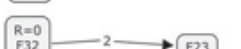 & $\stackrel{E=2}{\longrightarrow}-1 \longrightarrow$ & F43 & 0,09 & 0,00 & 0,09 & $-1,00$ \\
\hline$E=2$ & & F45 & 0,06 & 0,03 & 0,03 & $-1,00$ \\
\hline & & & w & lla & 0 & $\begin{array}{l}\text { al, de } \\
\text { ivo }\end{array}$ \\
\hline
\end{tabular}

Ilustración 12. Sesión de Foro No. 5 Inferencia Lógica - Grupo Heterogéneo 
En el grafo de la ilustración 12, se aprecia que el tutor, nodo F23, tiene los índices más altos de liderazgo general, de grupo, de individuos y efectivo. Los nodos de los estudiantes tienen un liderazgo efectivo igual a cero o negativo, en todos los casos.

En síntesis, el liderazgo en la comunicación en el grupo de foro heterogéneo se caracteriza por:

- El tutor es el único actor del proceso de comunicación que presenta índices de liderazgo efectivo positivos.

- En los casos analizados, el tutor es también quien presenta los mayores índices de liderazgo general y de grupo, aunque en algunas sesiones algunos estudiantes presentan valores altos en estos indicadores.

- El liderazgo entre individuos sobresale especialmente entre los estudiantes, aunque los mensajes emitidos están dirigidos hacia el tutor, la interacción entre pares es nula.

\section{Síntesis del análisis de liderazgo por grupos}

\begin{tabular}{|c|c|c|c|c|}
\hline Grupo & $\begin{array}{c}\text { Liderazgo de } \\
\text { Grupo }\end{array}$ & Liderazgo General & $\begin{array}{l}\text { Liderazgo entre } \\
\text { individuos }\end{array}$ & Liderazgo Efectivo \\
\hline CHAT & $\begin{array}{l}\text { El tutor presenta } \\
\text { los mayores } \\
\text { índices de } \\
\text { liderazgo de } \\
\text { grupo. }\end{array}$ & $\begin{array}{l}\text { En el grupo de chat } \\
\text { homogéneo, el tutor } \\
\text { sobresale en el } \\
\text { liderazgo general, } \\
\text { mientras, en el grupo } \\
\text { de chat heterogéneo, } \\
\text { el tutor es superado } \\
\text { en el liderazgo } \\
\text { general por uno de } \\
\text { los estudiantes. }\end{array}$ & $\begin{array}{l}\text { Sobresale } \\
\text { especialmente entre } \\
\text { los estudiantes, } \\
\text { aunque los mensajes } \\
\text { emitidos están dirigidos } \\
\text { hacia el tutor. }\end{array}$ & $\begin{array}{l}\text { El tutor es el único actor del } \\
\text { proceso de comunicación } \\
\text { que presenta índices de } \\
\text { liderazgo efectivo positivos. }\end{array}$ \\
\hline & $\begin{array}{l}\text { En el grupo } \\
\text { homogéneo los } \\
\text { valores más altos } \\
\text { de liderazgo } \\
\text { de grupo se } \\
\text { distribuyen } \\
\text { entre el tutor y } \\
\text { algunos de los } \\
\text { estudiantes. }\end{array}$ & $\begin{array}{l}\text { En el grupo } \\
\text { homogéneo los } \\
\text { valores más altos de } \\
\text { liderazgo general se } \\
\text { distribuyen entre el } \\
\text { tutor y algunos de los } \\
\text { estudiantes. }\end{array}$ & $\begin{array}{l}\text { Sobresale } \\
\text { especialmente entre } \\
\text { los estudiantes, } \\
\text { aunque los mensajes } \\
\text { emitidos están dirigidos } \\
\text { en la mayoría de los } \\
\text { casos hacia el tutor, la } \\
\text { interacción entre pares } \\
\text { es nula. }\end{array}$ & $\begin{array}{l}\text { En el grupo homogéneo } \\
\text { los valores de liderazgo } \\
\text { efectivo son iguales a cero o } \\
\text { negativos tanto para el tutor } \\
\text { como para los estudiantes, } \\
\text { sólo en uno de los casos } \\
\text { el tutor presentó liderazgo } \\
\text { efectivo positivo. }\end{array}$ \\
\hline
\end{tabular}




\begin{tabular}{|l|l|l|l|l|}
\hline Grupo & $\begin{array}{c}\text { Liderazgo de } \\
\text { Grupo }\end{array}$ & Liderazgo General & $\begin{array}{c}\text { Liderazgo entre } \\
\text { individuos }\end{array}$ & \multicolumn{1}{|c|}{ Liderazgo Efectivo } \\
\hline FORO & $\begin{array}{l}\text { En el grupo } \\
\text { heterogéneo, el } \\
\text { tutor es quien } \\
\text { presenta los } \\
\text { mayores índices } \\
\text { de liderazgo de } \\
\text { grupo, aunque } \\
\text { en algunas } \\
\text { sesiones algunos } \\
\text { estudiantes } \\
\text { presentan valores }\end{array}$ & $\begin{array}{l}\text { En el grupo } \\
\text { hes quien presenta } \\
\text { los mayores índices } \\
\text { de liderazgo general, } \\
\text { aunque en algunas } \\
\text { sesiones algunos } \\
\text { estudiantes presentan } \\
\text { valores altos en este } \\
\text { indicador. }\end{array}$ & & $\begin{array}{l}\text { En el grupo heterogéneo, } \\
\text { el tutor es el único actor del } \\
\text { proceso de comunicación } \\
\text { que presenta índices de } \\
\text { liderazgo efectivo positivos. }\end{array}$ \\
& & & \\
\hline indicador. & & & \\
\hline
\end{tabular}

\section{CONCLUSIONES}

De acuerdo con los datos obtenidos, el escenario de comunicación de foro o chat no incide de manera consistente en la concentración del grupo en la solución de problemas ni en el mayor o menor porcentaje de emisión de mensajes relacionados con el clima social.

Las diferencia más significativa que se evidenció entre los escenarios de foro y chat se encuentra en la tasa de liderazgo del tutor, que en el foro es comparativamente más baja que en el chat. Esto pude explicarse por la calidad de las respuestas del foro, que son más elaboradas; sin embargo, se observó poco feedback, lo cual significaría que debe haber otro tipo de interacciones que no se observan directamente en el escenario de aula virtual, a partir de las cuales se construye el conocimiento por parte de los estudiantes.

En el foro, la tasa de respuesta es baja comparada con la del chat, fenómeno que se visualiza con los valores bajos de liderazgo efectivo.

En los grupos homogéneos, el liderazgo se distribuye de manera similar entre los participantes, excepción hecha del tutor. En los heterogéneos el liderazgo global, el individual o el liderazgo de grupo tiende a ser distribuido entre el tutor y uno o dos estudiantes que le compiten; sin embargo el índice de liderazgo efectivo es superior 
para el tutor. Esta conclusión concuerda con los resultados de investigaciones previas y su explicación puede encontrarse en los trabajos de McFadzean \& McKenzie (2001) que en grupos heterogéneos hallaron que los estudiantes escriben sus comentarios con mayor cuidado con el fin de asegurar que sean entendidos como es deseado, activando la participación para explicar puntos de vista con más deliberación

Se presenta como interesante para futuros proyectos analizar el liderazgo comunicativo en grupos en los cuales no participe el tutor, de modo que pueda identificarse el desarrollo del liderazgo entre los estudiantes sin la característica inhibitoria que genera una figura de autoridad como el tutor (Stephan \& Stephan, 2001). Igualmente analizar la satisfacción dentro del grupo, pues esta se asocia al proceso de participación y a la eficacia del aprendizaje colectivo como un alto predictor de éxito (George, \& Nunamaker, 1990, Tyran, 1997; citado en Lim J. \& Zhong, Y., 2006, p. 60) y además incide en la calidad de las contribuciones y en la relevancia con respecto al total de contribuciones en el proceso de colaboración (Tyran, 1997 citado por Lim J. \& Zhong, Y., 2006, p. 60).

\section{NOTAS}

1 Resultados de la investigación "Sincronía y conocimientos previos: efectos sobre el aprendizaje y consolidación de grupos en aulas virtuales", cofinanciada por, COLCIENCIAS, y UNAD, contrato 192 de 2005 código 1156-11-17709. Equipo de Investigación: Luis Facundo Maldonado Granados, Linda Alejandra Leal Urueña, Milcon Montenegro Gamba, Carlos Alberto Lineros González, Jairo Pérez Rubio, Julián Urrea Beltrán. Tutores: Patricia Leguizamón y Pedro José Carrillo.

\section{REFERENCIAS BILIOGRÁFICAS}

George, J. F.; Easton, G. K.; Nunamaker, J. F.; Northcraft, G. B. (1990). A study of collaborative group work with and without computer-based support. Information Systems Research, 11, 4, (394-415).

Lim, J.; Zhong, Y. (2006). The Interaction and Effects of Perceived Cultural Diversity, Group Size, Leadership, and Collaborative Learning Systems: An Experimental Study. Information
Resources Management Journal. Hershey: Oct-Dec. Vol. 19, Iss. 4, (5671).

Maldonado, L.; Leal, L.; Montenegro, M.; Pérez, J.; Lineros, C. (2005). Solución de problemas y consolidación de grupos: Incidencia del número de participantes en un ambiente de chat. Informe de Investigación. UNAD.

Mcfadzean, E.; Mckenzie, J. (2001). Facilitating virtual learning groups: 
A practical approach. The Journal of Management Development. Bradford. Vol. 20, Iss. 5/6; (470).

Ming, M. C. (2000). Effects of status on solutions, leadership, and evaluations during group problem solving. Sociology of Education. Albany. Vol. 73, Iss. 3, (175195).

Stephan, W. G.; Stephan, C. W. (2001). Improving intergroup relations. CA: Sage.
Sowa, J. F. (2000). Knowledge Representation. Logical, Philosophical, and Computational Foundations. Pacific Grove: Brooks/Cole.

Tyran, C.K. (1997). GSS to support classroom discussion. In Proceedings of the 3oth Hawaii International Conference on System Sciences. Los Alamitos: IEEE Press.

\section{PERFIL ACADÉMICO Y PROFESIONAL DE LOS AUTORES}

Luis Facundo Maldonado G. Ph D. en Sistemas aplicados a Educación. Investigador en Tecnologías de la Información aplicadas al aprendizaje autónomo, gestión de conocimiento y redes de sociales en educación. Profesor universitario en pregrado, magíster y doctorado. Coordinador Nacional del Sistema de Investigación de la Universidad Nacional Abierta y a Distancia.

E-mail: lufamagr@gmail.com

Linda Alejandra Leal Urueña. Ingeniera de Sistemas de la Universidad Nacional de Colombia, candidata a Magister en Tecnologías de la Información aplicadas a la Educación de la Universidad Pedagógica Nacional. Líder del grupo de investigación EVED e investigadora del grupo TECNICE. Tutora de la Escuela de Ciencias Básicas, Tecnología e Ingeniería de la UNAD.

E-mail: lindalealu@gmail.com

Milcon Montenegro Gamba. Ingeniero de Sistemas de la Universidad Nacional de Colombiainvestigador de los grupos EVED y TECNICE. Tutor de la Escuela de Ciencias Básicas, Tecnología e Ingeniería de la UNAD.

E-mail: milconmontenegro@gmail.com 


\section{DIRECCIÓN DE LOS AUTORES}

Calle 14 sur 14-23.

Oficina Sistema Nacional de InvestigaciónUNAD.

Bogotá (Colombia)

Fecha de recepción del artículo: 17/07/08

Fecha de aceptación del artículo: 10/02/09 